Article

\title{
Laparoscopic Surgery for Intrahepatic Cholangiocarcinoma: A Focus on Oncological Outcomes
}

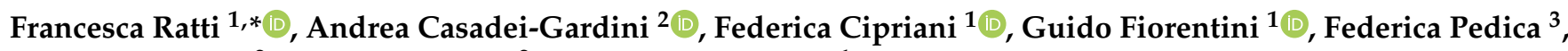 \\ Valentina Burgio ${ }^{2}$, Stefano Cascinu ${ }^{2}$ and Luca Aldrighetti ${ }^{1}$ \\ 1 Hepatobiliary Surgery Division, IRCCS San Raffaele Hospital, 20132 Milano, Italy; \\ cipriani.federica@hsr.it (F.C.); fiorentini.guido@hsr.it (G.F.); aldrighetti.luca@hsr.it (L.A.) \\ 2 Department of Medical Oncology, IRCCS San Raffaele Hospital, 20132 Milano, Italy; \\ casadeigardini@gmail.com (A.C.-G.); burgio.valentina@hsr.it (V.B.); cascinu.stefano@hsr.it (S.C.) \\ 3 Department of Experimental Oncology, Pathology Unit, San Raffaele Hospital, 20132 Milano, Italy; \\ pedica.federica@hsr.it \\ * Correspondence: ratti.francesca@hsr.it; Tel.: +39-02-26435778 (ext. 7808) or +39-348-2411961; \\ Fax: +39-02-26437807
}

check for

updates

Citation: Ratti, F.; Casadei-Gardini, A.; Cipriani, F.; Fiorentini, G.; Pedica, F.; Burgio, V.; Cascinu, S.; Aldrighetti, L. Laparoscopic Surgery for Intrahepatic Cholangiocarcinoma: A Focus on Oncological Outcomes. J. Clin. Med. 2021, 10, 2828. https:// doi.org/10.3390/jcm10132828

Academic Editor: Hauke Lang

Received: 5 May 2021

Accepted: 23 June 2021

Published: 26 June 2021

Publisher's Note: MDPI stays neutral with regard to jurisdictional claims in published maps and institutional affiliations.

Copyright: (c) 2021 by the authors. Licensee MDPI, Basel, Switzerland. This article is an open access article distributed under the terms and conditions of the Creative Commons Attribution (CC BY) license (https:/ / creativecommons.org/licenses/by/ $4.0 /)$.

\begin{abstract}
Background: The aim of the present study was to analyze the long-term outcomes of laparoscopic and open surgery for intrahepatic cholangiocarcinoma (iCCA) in a series, collected in a tertiary referral center with a high annual volume of laparoscopic activity. Methods: Between January 2004 and June 2020, 446 liver resections (LR) were performed for iCCA: of these, 179 were performed by laparoscopic surgery (LS) and 267 with the open approach. The two groups were matched through a 1:1 propensity score using covariates representative of patient and disease characteristics. The study and control groups were compared, with specific attention given to oncological outcomes (rate of R0, depth of resection margins, overall and disease-free survival, rate, and site of recurrence). Results: The number of retrieved nodes, rate, and depth of negative resection margins were comparable between the two groups. The interval time between surgery and subsequent adjuvant treatments was significantly shorter in LS patients. No differences were shown even in the comparison between the LS and the open group in terms of median disease-free and overall survival. Moreover, the disease recurrence rate was comparable between the LS and the open groups (45.2\% versus $56.7 \%$ ), and the recurrence pattern was similar. Conclusions: The minimally invasive approach for iCCA was once again confirmed to be associated with advantages in terms of intraoperative and short-term outcomes, but was also proven to be oncologically non-inferior to the open counterpart. In the present study, overall and disease-free survival were found to be similar between the two approaches.
\end{abstract}

Keywords: laparoscopy; minimally invasive; intrahepatic cholangiocarcinoma; new technique; liver resection

\section{Introduction}

Surgery is the only potentially curative treatment for intrahepatic cholangiocarcinoma (iCCA); the prerequisites-to be effective and associated with a long-term advantage for patients compared to non-curative treatments-include the radical excision of the disease with negative margins (frequently requiring major hepatectomies) and the removal of the locoregional lymph nodes of stations 8 and 12 [1-5]. These features are among the reasons why cholangiocarcinoma was later analyzed and described in the context of minimally invasive surgery [6-8] compared to other malignant tumors of the liver, such as hepatocellular carcinoma $[9,10]$ and colorectal liver metastases [11,12]. Indeed, indications of the role of laparoscopy in this area were not provided in the Southampton European consensus guidelines [13], while the literature has been enriched only in recent years with progressively larger series and studies comparing minimally invasive approaches with the open technique for iCCA [14,15]. Recently, therefore, reduced blood loss and 
blood transfusions, reduced postoperative morbidity, and faster postoperative recovery compared to the open technique are some of the advantages that have also been confirmed in the laparoscopic surgery of iCCA $[6-8,14,15]$.

Furthermore, the keypoints of iCCA surgery-initially deemed to be the most critical issues for initiating the large-scale diffusion of this technique-were specifically targeted to exclude their role as a real limit to the implementation of the laparoscopic technique. In particular, hot topics that deserved dedicated insight were the technical feasibility of major hepatectomies for large ICC nodules [16] and the adequacy of laparoscopic lymphadenectomy compared to the traditional open technique [7]. Indeed, the indisputable short-term advantages conferred by minimal invasiveness can avoid clashing with the achievement of adequate radicality, therefore jeopardizing the oncological efficacy of surgical treatment.

While for hepatocellular carcinoma and colorectal metastases the non-inferiority of the laparoscopic technique compared to the open one in terms of long-term outcomes is now a consolidated finding [9-13], the relative delay in the spread of laparoscopy for iCCA has resulted in a paucity of data regarding the comparative long-term results of using the open and laparoscopic approaches for this disease.

The primary endpoint of this study is therefore to evaluate, in a comparison between groups after a 1:1 propensity score matching, the long-term outcomes of laparoscopic and open surgery for iCCA in a series collected in a tertiary referral center with a high annual volume of laparoscopic activity. Our secondary purpose is to evaluate any differences conferred by the technical approach in intraoperative and short-term postoperative outcomes.

\section{Methods}

\subsection{Study Design}

The clinical, pathological, and short-term and long-term outcome data of patients undergoing surgical treatment for iCCA at the Hepatobiliary Surgery Division, San Raffaele Hospital, Milan, were prospectively collected and then retrospectively analyzed for the purposes of this study.

Patients with the following characteristics were excluded from the analysis: patients with Klatskin tumors or periductal infiltrating and intraductal cholangiocarcinomas, gallbladder cancers, unconfirmed iCCA on final pathology report, and less than 6 months of follow up. Between January 2004 and June 2020, 446 liver resections (LR) were performed for iCCA, of which 179 were performed using laparoscopic surgery (LS) and 267 with the open approach. The two groups were matched through a 1:1 propensity score using covariates representative of patient and disease characteristics. In this way, the study group (LS Group, $n=150$ ) and the control group (open group, $n=150$ ) were obtained. To fulfil the main endpoint, the study and control groups were compared, with specific attention given to oncological outcomes (rate of R0, depth of resection margins, overall and disease-free survival, rate and site of recurrence). The study design is shown in Figure 1. The standard implementation of the laparoscopic approach for iCCA was performed in our center between 2014 and 2015. Following this period, patients were standardly recruited to the laparoscopic approach unless presenting with one of the following characteristics: lesions requiring biliary or vascular resections; patients with lesions infiltrating the inferior vena cava; patients with lesions in contact with the hepatic vein of the future liver remnant (if only one vein was remaining in the remnant liver).

\subsection{Treatment Strategy and Surgical Technique}

Before surgery, all patients were evaluated by thoracoabdominal imaging (computed tomography and magnetic resonance imaging) and blood tests, including serum concentrations of tumor markers (carcinoembryonic antigen, Ca 19.9). Selected patients also underwent positron emission tomography (PET) imaging to rule out the presence of extrahepatic disease. Treatment strategies were routinely evaluated at weekly multidisciplinary 
meetings, during which liver surgeons, radiologists, hepatologists, and medical oncologists defined the indications for surgical procedures as well as the type and technique of resection [17].

Open approach-Under general anesthesia, xipho-supraumbilical incision extending to the right flank was performed in patients undergoing open LR.

Laparoscopic approach-Under general anesthesia, with the patient's legs apart and the first surgeon standing between them with one assistant at each side, a five-ports standard configuration (inverted J shape) was used.

In both the open and LS approaches, intraoperative ultrasound was performed routinely to assess the liver anatomy and to confirm resectability and the relationship between the lesion and major liver structures. Liver resection was performed by alternating the use of the ultrasonic dissector (Sonosurg, Olympus, Tokyo, Japan) and bipolar forceps, exposing the vascular structures, then coagulating or selectively sealing with clips or staplers, depending on the size. A formal lymphadenectomy was performed involving the complete removal of lymph nodes of stations 8 (on the common hepatic artery) and 12 (including regional nodes 12a along the hepatic artery, $12 \mathrm{~b}$ along the bile duct, and $12 p$ behind the portal vein) [7]. The Pringle maneuver was used as needed to control intraoperative bleeding.

Histological staging was performed according to the TNM classification following the criteria of the Seventh Edition of the American Joint Committee on Cancer (AJCC) [18]. R0 resection was defined as the absence of tumor cells on resection margins.

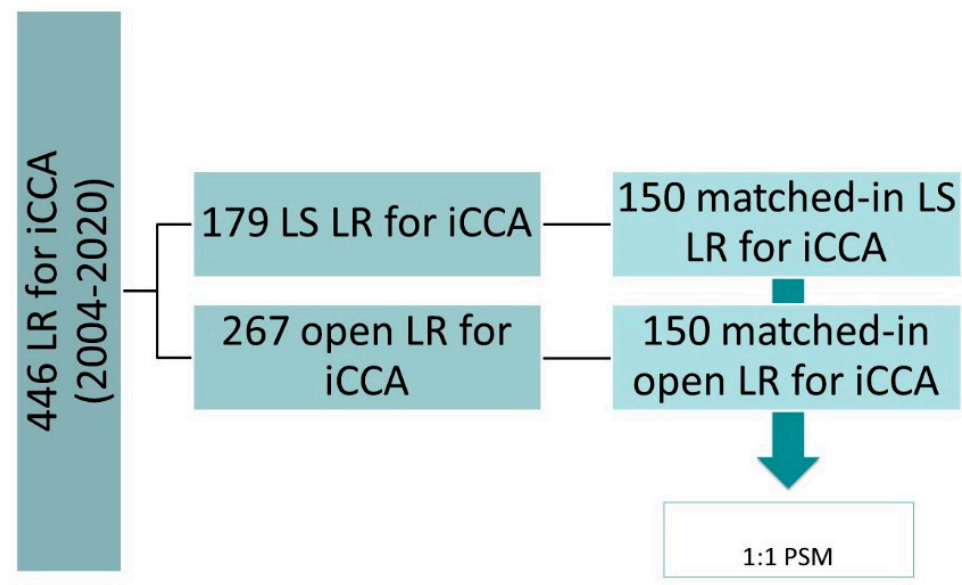

Figure 1. Study design. Abbreviations: iCCA, intrahepatic cholangiocarcinoma; LS, laparoscopic surgery; LR, liver resection; PSM, propensity score matching.

\subsection{Data Collection and Outcome Evaluation}

Data regarding the patient's preoperative and disease characteristics were collected, as well as intraoperative and pathological findings. Satellites are defined are lesions in close proximity to the primary tumor mass, while multiple lesions are those showing tumors arising in distant segments. The oncological risk of short-term recurrence was calculated for all the patients, using the postoperative prediction model of very early recurrence (VER) after hepatectomy for ICC (available at https:/ / k-sahara.shinyapps.io/Veryearlyrecurrence/ (accessed on 10 April 2021).

The overall complication rate at 90 days was reviewed and evaluated and complications were classified according to the Dindo-Clavien classification [19]. Mortality was defined as any death during the postoperative hospital stay or within 90 days of resection. The length of hospital stay and the time to full functional recovery were recorded and analyzed. Functional recovery was defined as the achievement of the following: adequate oral feeding, adequate pain control with oral analgesics, normal deambulation and self-care autonomy, and no complications. Data regarding follow-up, survival status, and occur- 
rence and type of relapse were analyzed. Three- and five-year overall survival (OS) and disease-free survival (DFS) were assessed using the Kaplan-Meier method.

\subsection{Statistical Methods}

To minimize the effect of bias and ensure the highest possible level of evidence in the context of a retrospective study, a 1:1 propensity score matching with a small caliper (0.2) was performed, taking into consideration all covariates which might have affected the selection and indication to the LS or open approach or the oncological outcomes. Those covariates included age, ASA (American Society of Anesthesiology) score, chronic liver disease (presence/absence), Ca 19.9 serum level, tumor dimension at preoperative imaging, number of lesions, and tumor stage. After matching, all variables were compared using the $\chi^{2}$ or Fisher's exact test for categorical data, the Mann-Whitney U test for nonnormally distributed continuous data, and the Student's $t$-test for normally distributed continuous variables. All data were expressed as the mean plus or minus the standard deviation or median and range. Survival curves were generated and compared using the Kaplan-Meier method. Significance was defined as $p<0.05$. All analyses were performed using the statistical package SPSS 26.0 (SPSS, Chicago, IL, USA).

\section{Results}

\subsection{Descriptive Data}

Patients and disease characteristics after propensity score matching are reported in Table 1. Age, sex, biometrics, and comorbidities were similar between the LS and the open groups. Underlying liver impairment or cirrhosis secondary to viral infection and/or alcohol-related damage was present in $30 \%$ of patients in the LS and $24 \%$ in the open group $(p=n s)$. All patients with liver impairment or cirrhosis had preserved liver function (Child A). Forty-four patients in the LS group had previous abdominal surgery (either open or laparoscopic) in their medical history. Most patients in both groups (72\% in the MILS and 70\% in the open group) had a single liver lesion. Lesion diameter was similar in the LS with the open series (respectively $5.3 \pm 2.3 \mathrm{~cm}$ versus $5.8 \pm 1.2 \mathrm{~cm}, p=\mathrm{ns}$ ). Eighty-eight percent of patients in the LS and $90 \%$ in the open group had intraoperative nodal dissection. Final pathological examination demonstrated nodal metastases in 56 out of $132(42.4 \%)$ patients in the MILS group and 53 of the 135 (39.2\%) patients who had undergone lymphadenectomy in the open group. Chemotherapy with neoadjuvant intent was administrated in a comparable proportion of patients in the laparoscopic compared with the open series (3.3\% versus $4.7 \%)$. The postoperative VER risk was low, intermediate, and high in $10.6 \%, 56.7 \%$, and $32.7 \%$ of patients in the LS group and $9.3 \%, 55.3 \%$, and $35.3 \%$ in the open group.

Table 1. Patients and disease characteristics after propensity score matching.

\begin{tabular}{|c|c|c|c|c|}
\hline & & LS Group $(n=150)$ & Open Group $(n=150)$ & $p$ \\
\hline Age (years) & Mean $\pm S D$ & $61 \pm 4$ & $62 \pm 7$ & ns \\
\hline Male sex, $n(\%)$ & & $92(61.3)$ & $86(57.3)$ & ns \\
\hline \multirow[t]{4}{*}{ ASA score, $n(\%)$} & & & & ns \\
\hline & 1 & $33(22)$ & $31(20.7)$ & \\
\hline & 2 & $79(52.7)$ & $83(55.3)$ & \\
\hline & 3 & $38(25.3)$ & $36(24)$ & \\
\hline BMI & Mean \pm SD & $24.6 \pm 2.6$ & $24.9 \pm 1.8$ & ns \\
\hline \multicolumn{2}{|c|}{ Underlying liver disease, $n(\%)$} & & & ns \\
\hline & None & $105(70)$ & $114(76)$ & \\
\hline & Steatosis/mild impairment & $25(16.7)$ & $23(15.3)$ & \\
\hline
\end{tabular}


Table 1. Cont.

\begin{tabular}{|c|c|c|c|}
\hline & LS Group ( $n=150)$ & Open Group $(n=150)$ & $p$ \\
\hline Cirrhosis & $20(13.3)$ & $13(8.7)$ & \\
\hline Previous abdominal surgery, $n(\%)$ & & & ns \\
\hline None & $107(71.3)$ & $118(78.7)$ & \\
\hline Yes, supramesocolic & $32(21.3)$ & $20(13.3)$ & \\
\hline Yes, inframesocolic & $12(8)$ & $12(8)$ & \\
\hline CEA (ng/mL) & $22 \pm 21$ & $35 \pm 19$ & ns \\
\hline Ca $19.9(\mathrm{U} / \mathrm{mL})$ & $89 \pm 76$ & $93 \pm 64$ & ns \\
\hline Mean \pm SD & $5.3 \pm 2.3$ & $5.8 \pm 1.2$ & ns \\
\hline Positive nodal status at imaging, $n(\%)$ & $17(11.3)$ & $32(21.3)$ & ns \\
\hline Tumor number, $n(\%)$ & & & ns \\
\hline Single & $108(72)$ & $105(70)$ & \\
\hline Single with satellites & $22(14.7)$ & $24(16)$ & \\
\hline Multiple & $20(13.3)$ & $21(14)$ & \\
\hline Histological grade, $n(\%)$ & & & ns \\
\hline Well & $20(13.3)$ & $12(8)$ & \\
\hline Moderate & 107 (71.3) & $122(81.3)$ & \\
\hline Poor & $25(16.7)$ & $16(10.7)$ & \\
\hline T stage, $n(\%) *$ & & & ns \\
\hline $\mathrm{T} 1 / \mathrm{T} 2$ & $111(74)$ & $114(76)$ & \\
\hline $\mathrm{T} 3 / \mathrm{T} 4$ & $39(26)$ & $36(24)$ & \\
\hline Lymphadenectomy, $n$ (\%) & $132(88)$ & $135(90)$ & ns \\
\hline Nodal status, $n(\%)$ & & & ns \\
\hline Negative & $94(62.7)$ & $97(64.7)$ & \\
\hline Positive & $56(37.3)$ & $53(35.3)$ & \\
\hline Staging, $n(\%) *$ & & & ns \\
\hline $\mathrm{I} / \mathrm{II}$ & $91(60.7)$ & $93(62)$ & \\
\hline III/IVa & $59(39.3)$ & $57(38)$ & \\
\hline Preoperative CT, $n(\%)$ & & & ns \\
\hline Yes & $5(3.3)$ & $7(4.7)$ & \\
\hline No & 145 (96.7) & $143(95.3)$ & \\
\hline Suspected need for vascular resection, $n(\%)$ & $2(1.3)$ & $3(2)$ & ns \\
\hline Suspected need for biliary resection, $n(\%)$ & $5(3.3)$ & $6(4)$ & ns \\
\hline VER Low risk, $n(\%)$ & $16(10.7)$ & $14(9.3)$ & ns \\
\hline VER Intermediate-risk, $n(\%)$ & $85(56.7)$ & $83(55.3)$ & ns \\
\hline VER High risk, $n(\%)$ & $49(32.7)$ & $53(35.3)$ & ns \\
\hline
\end{tabular}

Abbreviations: ASA, American Society of Anesthesiology; BMI, Body Mass Index; CA, carbohydrate antigen; CEA, carcinoembryonic antigen; T, tumor; CT, chemotherapy; VER, very early recurrence; ns, not significant. * Defined according to the 8 th Edition of the AJCC classification.

\subsection{Short-Term Outcome Data}

Operative characteristics are shown in Table 2. Major hepatectomies (removal of $\geq 3$ liver segments) were performed in 51 (34\%) (in detail: 24 right, 25 left, and 2 central 
hepatectomies) and 55 (36.7\%) (in detail: 26 right, 27 left and 2 central hepatectomies) patients in the MILS and open groups, respectively $(p=n s)$.

Table 2. Intra- and postoperative details. * Grade of complication was calculated according to the Dindo-Clavien classification of surgical complications [19].

\begin{tabular}{|c|c|c|c|c|}
\hline & & LS Group $(n=150)$ & Open Group $(n=150)$ & $p$ \\
\hline \multirow[t]{3}{*}{ Procedure, $n(\%) *$} & & & & ns \\
\hline & Minor resection & $99(66)$ & $95(63.3)$ & \\
\hline & Major resection & $51(34)$ & $55(36.7)$ & \\
\hline \multirow[t]{3}{*}{ Pringle Manuevre, $n(\%)$} & & & & ns \\
\hline & Not performed & $17(11.3)$ & $14(9.3)$ & \\
\hline & Performed & $133(88.7)$ & $136(90.7)$ & \\
\hline Lenght of surgery (min) & Mean \pm SD & $270 \pm 65$ & $230 \pm 60$ & ns \\
\hline Blood loss (mL) & Mean \pm SD & $150 \pm 100$ & $350 \pm 250$ & 0.021 \\
\hline Number of retrieved nodes & Median (range) & $9(5-11)$ & $7(5-14)$ & ns \\
\hline \multirow[t]{3}{*}{ Surgical margin, $n(\%)$} & & & & ns \\
\hline & R0 & $146(97.3)$ & $143(95.3)$ & \\
\hline & $\mathrm{R} 1$ & $4(2.7)$ & $7(4.7)$ & \\
\hline Conversion, $n(\%)$ & & $17(11.3)$ & n.a. & \\
\hline Surgical margin $(\mathrm{mm})$ & Mean \pm SD & $6 \pm 4$ & $7 \pm 4$ & ns \\
\hline \multirow[t]{3}{*}{ Intraoperative blood transfusions, $n(\%)$} & & & & 0.04 \\
\hline & No & $144(96)$ & $138(92)$ & \\
\hline & Yes & $6(4)$ & $12(8)$ & \\
\hline \multirow[t]{3}{*}{ Postoperative blood transfusions, $n(\%)$} & & & & 0.03 \\
\hline & No & $140(93.3)$ & $128(85.3)$ & \\
\hline & Yes & $10(6.7)$ & $22(14.7)$ & \\
\hline \multicolumn{5}{|l|}{ Complications, $n(\%)$} \\
\hline & Hemorrhage & 0 & $3(2)$ & ns \\
\hline & Wound infection & $2(1.3)$ & $6(4)$ & 0.02 \\
\hline & Ileus & $2(1.3)$ & $7(4.7)$ & 0.01 \\
\hline & Biliary fistula & $6(4)$ & $12(8)$ & 0.03 \\
\hline & $\begin{array}{l}\text { Transient liver } \\
\text { failure }\end{array}$ & $4(2.7)$ & $7(4.7)$ & ns \\
\hline & Ascites & $10(6.7)$ & $16(10.7)$ & 0.03 \\
\hline & Pleural effusion & $10(6.7)$ & $14(9.3)$ & 0.04 \\
\hline & Pneumonia & $2(1.3)$ & $4(2.7)$ & ns \\
\hline & Fever & $6(4)$ & $9(6)$ & ns \\
\hline & Pancreatitis & $3(2)$ & $3(2)$ & ns \\
\hline & Lymphatic fistula & $3(2)$ & $10(6.7)$ & 0.03 \\
\hline & Arrhythmia & $4(2.7)$ & $6(4)$ & 0.04 \\
\hline & $\mathrm{DVT} / \mathrm{PE}$ & $2(1.3)$ & $3(2)$ & ns \\
\hline Overall complication rate, $n(\%)$ & & $22(14.7)$ & $36(24)$ & 0.015 \\
\hline Grade of complications, $n(\%) *$ & & & & 0.03 \\
\hline
\end{tabular}


Table 2. Cont.

\begin{tabular}{|c|c|c|c|c|}
\hline & & LS Group ( $n=150)$ & Open Group $(n=150)$ & $p$ \\
\hline \multirow[t]{2}{*}{ Minor } & I grade & $4(2.7)$ & $7(4.7)$ & \\
\hline & II grade & $12(8)$ & $17(11.3)$ & \\
\hline \multirow[t]{2}{*}{ Major } & III grade & $4(2.7)$ & $9(6)$ & ns \\
\hline & IV grade & $2(1.3)$ & $3(2)$ & \\
\hline Mortality, $n(\%)$ & & $2(1.3)$ & $2(1.3)$ & ns \\
\hline Functional recovery (days) & Median (range) & $3(1-5)$ & $5(3-10)$ & 0.032 \\
\hline Lenght of stay (days) & Median (range) & $4(2-10)$ & $6(3-21)$ & 0.046 \\
\hline Interval surgery-adjuvant treatment (days) & Median (range) & $35(30-55)$ & $49(37-63)$ & 0.03 \\
\hline Systemic adjuvant therapy, $n(\%)$ & & $124(82.7)$ & $116(77.3)$ & 0.05 \\
\hline
\end{tabular}

Abbreviations: DVT, deep vein thrombosis; PE, pulmonary embolism; ns, not significant.

The length of surgery was comparable between the two groups. Laparoscopic procedures were successfully completed in 133 patients, whereas the procedure was converted to laparotomy in 17 patients. Concerns regarding oncologic inadequacy (10 cases), bleeding (6 cases), and adhesions (1 case) were the causes of conversion. Significantly reduced intraoperative blood loss was recorded in the LS (mean $150 \pm 100 \mathrm{~mL}$ ) compared with the open group (mean $350 \pm 250 \mathrm{~mL}$ ) with $p=0.021$, despite the comparable intraoperative use of the Pringle maneuver between the two groups $(88.7 \%$ in the LS and $90.7 \%$ in the open group).

The median number of retrieved nodes, the achievement of negative resection margins, and the depth of surgical margins on liver parenchyma were comparable between both groups.

Postoperative overall complications rate and mortality are reported in Table 2. Postoperative complications occurred in $14.7 \%$ of patients in the LS group and in $24 \%$ of patients in the open group $(p=0.015)$. In particular, the incidence of minor (Dindo-Clavien $<3$ ) complications was significantly lower in the LS compared with the open group (10.7\% versus $16 \%$, respectively; $p=0.03$ ). The benefit in terms of reduced incidence of complications was evident when specifically analyzing the incidence in the LS compared with the open group in terms of wound infection, postoperative ileus, biliary fistula, ascites, pleural effusion, lymphatic fistula, and arrhythmia.

The LS group, compared with the open group, showed a significant reduction in both median hospital stay and time for functional recovery ( 3 and 5 days, $p=0.032$ and 4 and 6 days, $p=0.046$ respectively). Furthermore, the interval time between surgery and subsequent adjuvant treatments ( 35 and 49 days median in the LS and the open group, respectively; $p=0.03$ ) was significantly shorter in LS patients. A higher number of patients in the LS compared with the open group $(82.7 \%$ and $77.3 \%)$ received systemic adjuvant therapies, without reaching statistical significance.

\subsection{Long-Term Outcome Data}

The median disease-free survival was 28 months (range 26-30) in the open and 32 months (range 28-35) in the LS group, with no significant difference. No differences were shown even in the comparison between the LS and the open group in terms of median overall survival (44 months, range 37-45 and 41 months, range 33-44, respectively). Kaplan-Meier curves for disease-free and overall survival according to the treatment group are shown in Figure 2a,b, respectively. Table 3 reports the incidence of recurrence and patterns of recurrence in the study and control groups. In particular, the disease recurrence rate was comparable in the LS compared with the open group ( $45.2 \%$ versus $56.7 \%)$, and the recurrence pattern was similar. Recurrence at the site of the resection margin, peritoneal seeding, or port-site metastases were not observed in any patients in the LS group. The treatment of recurrences was similar between the groups. 

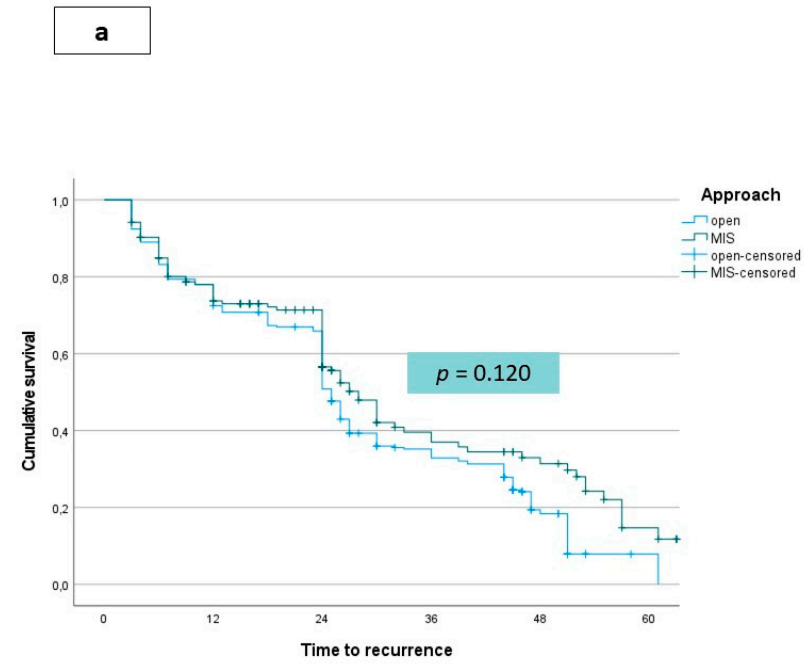

b

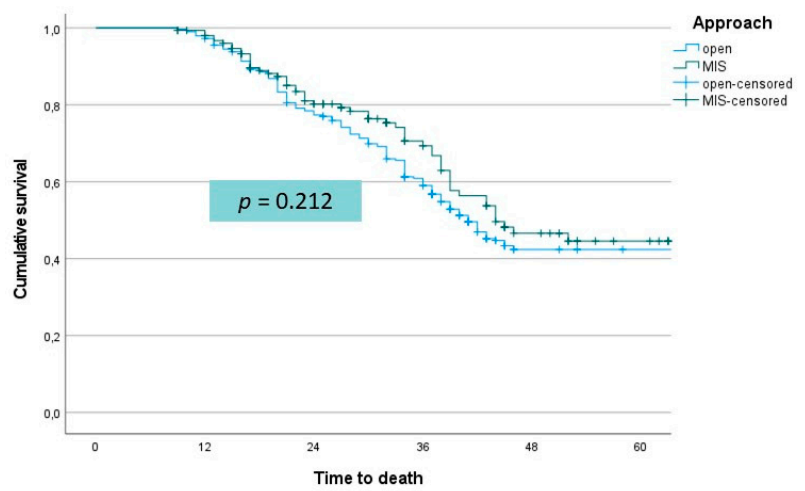

Figure 2. Survival functions. (a) Disease free survival according to treatment group (open versus laparoscopic). (b) Overall survival according to treatment group (open versus laparoscopic).

Table 3. Long-term outcomes.

\begin{tabular}{|c|c|c|c|c|}
\hline & & LS Group $(n=150)$ & Open Group $(n=150)$ & $p$ \\
\hline Disease recurrence $(n) \%$ & & $89(59.3)$ & $95(63.3)$ & ns \\
\hline \multirow[t]{5}{*}{ Modality of recurrence, $n(\%) *$} & & & & $\mathrm{~ns}$ \\
\hline & Nodal & $17(19.1)$ & $19(20)$ & \\
\hline & Intrahepatic, monofocal & $19(21.3)$ & $18(18.9)$ & \\
\hline & Intrahepatic, multifocal & $47(52.8)$ & $51(53.7)$ & \\
\hline & Extrahepatic & $26(29.2)$ & $31(32.6)$ & \\
\hline \multirow[t]{4}{*}{ Therapy of recurrence, $n(\%) *$} & & & & ns \\
\hline & Re-resection & $11(12.4)$ & $10(10.5)$ & \\
\hline & Medical therapy & $69(77.5)$ & $76(80)$ & \\
\hline & Other local treatments & $13(14.6)$ & $15(15.8)$ & \\
\hline
\end{tabular}

* Percentage calculated on the number of patients who developed a recurrence of the disease.

\section{Discussion}

The relative delay in the diffusion of the minimally invasive approach for iCCA has resulted in the current scarce availability of data on the oncological outcomes of this technique compared to the traditional open one [14] The present study is, to the best of our knowledge, the first to deal specifically with this issue: in two groups of patients homogeneously selected by propensity score matching, the LS technique proved to be similar in terms of the 3- and 5- year disease-free and overall survival to the open group. The most consistent advantages of LS are: first, the limitation of biological stress ensured by laparoscopy contributing to the maintenance of immune competence [20], while, conversely, immunosuppression linked to significant surgical stress greatly reduces the ability to respond to tumorigenesis and consequently increases the neoplastic risk, primarily that of relapse $[21,22]$. Secondly, the laparoscopic approach, making the postoperative course more favorable and reducing the time required for functional recovery, allows patients to start systemic chemotherapy programs more promptly [11,23]. In fact, although the overall number of patients undergoing adjuvant chemotherapy program is comparable between the two groups, in the laparoscopic group there is a shorter median surgery-tostart chemotherapy interval and a significantly higher number of patients who have started the cancer program within two and three months from surgical treatment. 
The main challenge reported for the initial exclusion from minimally invasive programs of patients with iCCA is the need to perform a formal lymphadenectomy, which is required both for reasons related to the complete staging of the disease and for curative purposes $[6-8,15,16]$. Indeed, Bagante et al. reported a prognostic advantage from the removal of stations 8 and 12 during surgery [24]. The prerequisite for identifying an adequate lymphadenectomy is the removal of at least six lymph nodes [24,25]. In our previous experiments published in 2019, the effectiveness of laparoscopic compared to open lymphadenectomy in a mixed series of intrahepatic cholangiocarcinoma and gallbladder carcinoma was specifically analyzed. The number of lymph nodes collected was comparable in the laparoscopic and open series ( 8 versus 7 , respectively; $p=$ not significant); however, interestingly the percentage of patients who reached the AJCC recommended cut-off of six lymph nodes harvested was higher in the laparoscopic than in the open group. These results in the laparoscopic group were obtained without negatively affecting the overall postoperative morbidity and morbidity related to lymphadenectomy [7]. In a subsequent bi-institutional series including the results of a series of 208 patients, half of whom were treated by laparoscopic invasive approach and half by the open technique, the advantages of laparoscopy already described in other primary and secondary neoplasms of the liver were reconfirmed specifically in the iCCA, in a large series belonging to referral centers with HPB expertise [8]. In that series, the assessment of long-term outcomes was still affected by a relatively short observation period of the laparoscopic series, but a faster return to adjuvant programs had already been observed in the laparoscopic group [8]. In a recent meta-analysis by Regmi et al., eight studies were identified reporting the data of 552 patients operated on for laparoscopy and compared with 2320 open cases [26]. Considering data supporting the use of mini-invasiveness but still with significant limitations in terms of patients recruitment, the authors concluded that LS for iCCA is still in the early exploration phase, and consequently stronger evidence is needed to support its use and to draw conclusions, especially in terms of long-term outcomes [26].

The results of this series are important, since they not only confirm the non-inferiority of the laparoscopic technique in terms of long-term outcomes but also allow us to open a new scenario on the crucial value added by this technique in the natural history of patients with iCCA. In fact, patients with iCCA benefit from multimodal treatment, especially if negative prognostic factors such as multifocality and lymph node involvement are detected. Within a multidisciplinary discussion, the indication to start an adjuvant treatment is aimed to add a prognostic advantage in patients already treated with surgery. In the BILCAP study, a randomized, controlled, multicenter, phase 3 study conducted across 44 specialist hepatopancreatobiliary centers in the UK, patients were assigned to receive oral capecitabine versus observation [27]. In the per-protocol analysis, median recurrencefree survival was 25.9 months in the capecitabine group and 17.4 months in the observation group, strongly supporting the use of adjuvant therapies in resected patients. Interestingly, in this study patients who had not completely recovery from previous surgery were excluded and study eligibility to 16 weeks after surgery was allowed after two amendments (probably because too many patients needed to be excluded because of performance status in the interval of time closer to surgery) [27]. Consequently, there are no current data on the relationship between the timing of the initiation of chemotherapy and the impact on the risk of recurrence. However, it can be speculated that there is a correlation between early treatment initiation and oncological benefit. As already reported in other areas of oncological surgery of the liver, laparoscopy allows an earlier start of the adjuvant therapy also in cholangiocarcinoma [11,23]. Beyond the median surgery-adjuvant interval time, even the number of patients who had started the adjuvant treatment at two and three months after surgery were assessed; although the difference between the two approaches decreased over months, the LS group had a significantly higher number of cases with treatment initiation at both 2 and 3 months.

Furthermore, Sheka et al. reported that only $51 \%$ of patients who undergo up ront surgery actually receive NCCN-recommended adjuvant therapy [28]. It is therefore 
possible - and will be interesting to evaluate in the future- that the reduction in the biological impact of surgery allows a greater number of patients (who, due to the staging and characteristics of the disease, would have an indication for adjuvant therapies) to be able to start the scheduled program thanks to an adequate performance status. In fact, in Sheka et al. it was also reported how the receipt of surgery + chemotherapy was independently associated with survival on Cox proportional hazard ratio modeling compared to surgery alone or chemotherapy alone, and failure to administer multimodality therapy leads to sub-optimal outcomes for patients with node -positive biliary tract cancers [28].

This issue is remarkably relevant in patients with a high risk of VER. Tsimiligras et al. in fact reported that approximately one fourth of patients undergoing curative intent hepatectomy for iCCA develop VER, and that VER is associated with discouraging prognosis [29]. Obviously, in the presence of a very high preoperative risk of VER, the hypothesis of neoadjuvant chemotherapy must be considered by the multidisciplinary team. However, there might be a discrepancy between the preoperative and the postoperative risk score of VER, and this is not an infrequent situation. For instance, if a formal lymphadenectomy is performed allowing the identification of patients with positive lymph nodes even if they were not enlarged on CT scan or non-enhancing in PET, the postoperative score (i.e., the one used in this study) may indicate a high risk of VER, while the preoperative risk may be moderate. In these patients, the ability to start early treatment is crucial [29].

In conclusion, the laparoscopic approach for iCCA is once again confirmed to be associated with advantages in terms of intraoperative and short-term outcome, but also proves to be oncologically non-inferior to the open counterpart. In the present study, the overall and disease-free survival were found to be similar between the two. It is possible that laparoscopy can provide a specific benefit to patients with a higher risk of recurrence, for reasons that still need to be explained but that are likely related to the favorable biological scenario and to the possibility of starting the adjuvant chemotherapy program earlier.

Author Contributions: Conceptualization, F.R. and L.A.; methodology, A.C.-G.; software F.G.; C.F.; validation, L.A. and C.S.; formal analysis, P.F.; investigation, L.A., F.P.; resources, F.P.; data curation, F.C.; writing—original draft preparation, F.R.; writing — review and editing, L.A. and C.S.; visualization, F.G.; supervision, V.B.; project administration, B.V.; funding acquisition, L.A. All authors have read and agreed to the published version of the manuscript.

Funding: The research leading to these results has received funding from Fondazione AIRC under 5 per Mille 2019-ID. 22737 program-P.I. Bonini Maria Chiara, G.L. Aldrighetti Luca Antonio.

Institutional Review Board Statement: The study was conducted according to the guidelines of the Declaration of Helsinki, and approved by the Institutional Review Board of IRCCS San Raffaele Hospital.

Informed Consent Statement: Informed consent was obtained from all subjects involved in the study.

Data Availability Statement: The data presented in this study are available on request from the corresponding author. The data are not publicly available due to privacy reasons.

Conflicts of Interest: The material has not been previously published or submitted elsewhere for publication and will not be sent to another journal until a decision is made concerning publication All listed authors have participated in the study and have approved the final manuscript. There is no personal conflicts of interest or financial disclosure for any of the authors. The authors of this manuscript have no conflicts of interest to disclose and further disclose any commercial interest that they may have in the subject of study and the source of any financial or material support.

\section{References}

1. Weber, S.M.; Ribero, D.; O’Reilly, E.M.; Kokudo, N.; Miyazaki, M.; Pawlik, T.M. Intrahepatic Cholangiocarcinoma: Expert consensus statement. HPB (Oxford) 2015, 17, 669-680. [CrossRef]

2. Maithel, S.K.; Gamblin, T.C.; Kamel, I.; Corona-Villalobos, C.P.; Thomas, M.; Pawlik, T.M. Multidisciplinary approaches to intrahepatic cholangiocarcinoma. Cancer 2013, 119, 3929-3942. [CrossRef] 
3. Spolverato, G.; Bagante, F.; Ethun, C.G.; Poultsides, G.; Tran, T.; Idrees, K.; Isom, C.A.; Fields, R.C.; Krasnick, B.; Winslow, E.; et al. Defining the Chance of Statistical Cure Among Patients with Extrahepatic Biliary Tract Cancer. World J. Surg. 2017, 41, $224-231$. [CrossRef]

4. Sasaki, K.; Margonis, G.A.; Andreatos, N.; Bagante, F.; Weiss, M.; Barbon, C.; Popescu, I.; Marques, H.P.; Aldrighetti, L.; Maithel, S.K.; et al. Preoperative Risk Score and Prediction of Long-Term Outcomes after Hepatectomy for Intrahepatic Cholangiocarcinoma. J. Am. Coll. Surg. 2018, 226, 393-403. [CrossRef]

5. Ribero, D.; Pinna, A.D.; Guglielmi, A.; Ponti, A.; Nuzzo, G.; Giulini, S.M.; Aldrighetti, L.; Calise, F.; Gerunda, G.E.; Tomatis, M.; et al. Surgical approach for long-term survival of patients with intrahepatic cholangiocarcinoma: A multi-institutional analysis of 434 patients. Arch. Surg. 2012, 147, 1107-1113. [CrossRef] [PubMed]

6. Ratti, F.; Cipriani, F.; Ariotti, R.; Gagliano, A.; Paganelli, M.; Catena, M.; Aldrighetti, L. Safety and feasibility of laparoscopic liver resection with associated lymphadenectomy for intrahepatic cholangiocarcinoma: A propensity score-based case-matched analysis from a single institution. Surg. Endosc. 2016, 30, 1999-2010. [CrossRef]

7. Ratti, F.; Fiorentini, G.; Cipriani, F.; Paganelli, M.; Catena, M.; Aldrighetti, L. Perioperative and Long-Term Outcomes of Laparoscopic Versus Open Lymphadenectomy for Biliary Tumors: A Propensity-Score-Based, Case-Matched Analysis. Ann. Surg. Oncol. 2019, 26, 564-575. [CrossRef] [PubMed]

8. Ratti, F.; Rawashdeh, A.; Cipriani, F.; Primrose, J.; Fiorentini, G.; Abu Hilal, M.; Aldrighetti, L. Intrahepatic cholangiocarcinoma as the new field of implementation of laparoscopic liver resection programs. A comparative propensity score-based analysis of open and laparoscopic liver resections. Surg. Endosc. 2021, 35, 1851-1862. [CrossRef] [PubMed]

9. Serenari, M.; Ratti, F.; Zanello, M.; Guglielmo, N.; Mocchegiani, F.; Di Benedetto, F.; Nardo, B.; Mazzaferro, V.; Cillo, U.; Massani, M.; et al. Minimally Invasive Stage 1 to Protect Against the Risk of Liver Failure: Results from the Hepatocellular Carcinoma Series of the Associating Liver Partition and Portal Vein Ligation for Staged Hepatectomy Italian Registry. J. Laparoendosc. Adv. Surg. Tech. 2020, 30, 1082-1089. [CrossRef]

10. Ratti, F.; Cipriani, F.; Fiorentini, G.; Catena, M.; Paganelli, M.; Aldrighetti, L. Reappraisal of the advantages of laparoscopic liver resection for intermediate hepatocellular carcinoma within a stage migration perspective: Propensity score analysis of the differential benefit. J. Hepato-Biliary-Pancreat. Sci. 2020, 27, 510-521. [CrossRef]

11. Ratti, F.; Fiorentini, G.; Cipriani, F.; Catena, M.; Paganelli, M.; Aldrighetti, L. Laparoscopic vs Open Surgery for Colorectal Liver Metastases. JAMA Surg. 2018, 153, 1028-1035. [CrossRef] [PubMed]

12. Fretland, Å.A.; Dagenborg, V.J.; Bjørnelv, G.M.W.; Kazaryan, A.M.; Kristiansen, R.; Fagerland, M.W.; Hausken, J.; Tønnessen, T.I.; Abildgaard, A.; Barkhatov, L.; et al. Laparoscopic Versus Open Resection for Colorectal Liver Metastases The OSLO-COMET Randomized Controlled Trial. Ann. Surg. 2018, 267, 199-207. [CrossRef]

13. Abu H., M.; Aldrighetti, L.; Dagher, I.; Edwin, B.; Troisi, R.I.; Alikhanov, R.; Aroori, S.; Belli, G.; Besselink, M.; Briceno, J.; et al. The Southampton Consensus Guidelines for Laparoscopic Liver Surgery: From Indication to Implementation. Ann. Surg. 2018, $268,11-18$.

14. Machairas, N.; Kostakis, I.D.; Schizas, D.; Kykalos, S.; Nikiteas, N.; Sotiropoulos, G.C. Meta-analysis of laparoscopic versus open liver resection for intrahepatic cholangiocarcinoma. Updat. Surg. 2021, 73, 59-68. [CrossRef] [PubMed]

15. Ziogas, I.A.; Esagian, S.M.; Giannis, D.; Hayat, M.H.; Kosmidis, D.; Matsuoka, L.K.; Montenovo, M.I.; Tsoulfas, G.; Geller, D.A.; Alexopoulos, S.P. Laparoscopic versus open hepatectomy for intrahepatic cholangiocarcinoma: An individual patient data survival meta-analysis. Am. J. Surg. 2021, 26. [CrossRef]

16. Zhu, Y.; Song, J.; Xu, X.; Tan, Y.; Yang, J. Safety and feasibility of laparoscopic liver resection for patients with large or multiple intrahepatic cholangiocarcinomas: A propensity score based case-matched analysis from a single institute. Medicine 2019, 98, e18307. [CrossRef] [PubMed]

17. Ratti, F.; Cipriani, F.; Fiorentini, G.; Salinas, C.H.; Catena, M.; Paganelli, M.; Aldrighetti, L. Management of hilum infiltrating tumors of the liver: The impact of experience and standardization on outcome. Dig. Liver Dis. 2019, 51, 135-141. [CrossRef] [PubMed]

18. Juntermanns, B.; Kaiser, G.M.; Orth, L.; Reis, H.; Jaradat, D.; Sydor, S.; Buechter, M.; Kasper, S.; Mathe, Z.; Sotiropoulos, G.C.; et al. Comparison of the sixth and the seventh editions of the UICC classification for intrahepatic cholangiocarcinoma. Eur. J. Med Res. 2018, 23, 29. [CrossRef]

19. Dindo, D.; Demartines, N.; Clavien, P.A. Classification of surgical complications: A new proposal with evaluation in a cohort of 6336 patients and results of a survey. Ann. Surg. 2004, 240, 205-213. [CrossRef] [PubMed]

20. Fretland, A.A.; Sokolov, A.; Postriganova, N.; Kazaryan, A.M.; Pischke, S.; Nilsson, P.H.; Rognes, I.N.; Bjornbeth, B.A.; Fagerland, M.W.; Mollnes, T.E.; et al. Inflammatory Response After Laparoscopic Versus Open Resection of Colorectal Liver Metastases: Data From the Oslo-CoMet Trial. Medicine 2015, 94, e1786. [CrossRef]

21. Reames, B.N.; Bagante, F.; Ejaz, A.; Spolverato, G.; Ruzzenente, A.; Weiss, M.; Alexandrescu, S.; Marques, H.P.; Aldrighetti, L.; Maithel, S.K.; et al. Impact of adjuvant chemotherapy on survival in patients with intrahepatic cholangiocarcinoma: A multi-institutional analysis. HPB (Oxford) 2017, 19, 901-909. [CrossRef] [PubMed]

22. Zhou, G.; Sprengers, D.; Mancham, S.; Erkens, R.; Boor, P.P.; van Beek, A.A.; Doukas, M.; Noordam, L.; Carrascosa, L.C.; de Ruiter, V.; et al. Reduction of immunosuppressive tumor microenvironment in cholangiocarcinoma by ex vivo targeting immune checkpoint molecules. J. Hepatol. 2019, 71, 753-762. [CrossRef] 
23. Ratti, F.; Fuks, D.; Cipriani, F.; Gayet, B.; Aldrighetti, L. Timing of Perioperative Chemotherapy Does Not Influence LongTerm Outcome of Patients Undergoing Combined Laparoscopic Colorectal and Liver Resection in Selected Upfront Resectable Synchronous Liver Metastases. World J. Surg. 2019, 43, 3110-3119. [CrossRef] [PubMed]

24. Bagante, F.; Gani, F.; Spolverato, G.; Xu, L.; Alexandrescu, S.; Marques, H.P.; Lamelas, J.; Aldrighetti, L.; Gamblin, T.C.; Maithel, S.K.; et al. Intrahepatic Cholangiocarcinoma: Prognosis of Patients Who Did Not Undergo Lymphadenectomy. J. Am. Coll. Surg. 2015, 221, 1031-1040. [CrossRef] [PubMed]

25. Bagante, F.; Spolverato, G.; Weiss, M.; Alexandrescu, S.; Marques, H.P.; Aldrighetti, L.; Maithel, S.K.; Pulitano, C.; Bauer, T.W.; Shen, F; et al. Assessment of the Lymph Node Status in Patients Undergoing Liver Resection for Intrahepatic Cholangiocarcinoma: The New Eighth Edition AJCC Staging System. J. Gastrointest. Surg. 2018, 22, 52-59. [CrossRef] [PubMed]

26. Regmi, P.; Hu, H.-J.; Paudyal, P.; Liu, F.; Ma, W.-J.; Yin, C.-H.; Jin, Y.-W.; Li, F.-Y. Is laparoscopic liver resection safe for intrahepatic cholangiocarcinoma? A meta-analysis. Eur. J. Surg. Oncol. (EJSO) 2020, 47, 979-989. [CrossRef]

27. Primrose, J.N.; Fox, R.P.; Palmer, D.H.; Malik, H.Z.; Prasad, R.; Mirza, D.; Anthony, A.; Corrie, P.; Falk, S.; Finch-Jones, M.; et al. Capecitabine compared with observation in resected biliary tract cancer (BILCAP): A randomised, controlled, multicentre, phase 3 study. Lancet Oncol. 2019, 20, 663-673. [CrossRef]

28. Sheka, A.C.; Altman, A.; Marmor, S.; Hui, J.Y.; Denbo, J.W.; Ankeny, J.S.; Jensen, E.H. Failure to administer multimodality therapy leads to sub-optimal outcomes for patients with node-positive biliary tract cancers in the United States. Surg. Oncol. 2020, 34, 298-303. [CrossRef]

29. Tsilimigras, D.I.; Sahara, K.; Wu, L.; Moris, D.; Bagante, F.; Guglielmi, A.; Aldrighetti, L.; Weiss, M.; Bauer, T.W.; Alexandrescu, S.; et al. Very Early Recurrence After Liver Resection for Intrahepatic Cholangiocarcinoma: Considering Alternative Treatment Approaches. JAMA Surg. 2020, 155, 823. [CrossRef] [PubMed] 